\title{
Redeploying plant defences
}

\author{
Epidemic diseases are not a new phenomenon, but easy access to transport in the modern world has accelerated \\ their spread. Perhaps some botanical understanding can help slow them down.
}

T's:

he ongoing outbreak of COVID-19, caused by the coronavirus SARSCoV-2, originated in Wuhan city, China, last December. Hitchhiking on the Spring Festival travel season, the rapid and highly infectious virus quickly spread around the country and has become a global problem. Tens of thousands of people, including many medical workers, have been infected, and thousands have died. Due to an incubation period of 14 days or longer, patients can cause multiple infections before symptoms become observable. An effective cure is yet to be developed.

The complicated secondary metabolism of plants has been the source of countless medicinal compounds and leads for drug discovery. It is little surprise then that plant products and their analogues have been employed as an early line of defence against COVID-19. On 17 February, the Chinese State Council announced that chloroquine phosphate - a structural analogue of quinine, originally extracted from the bark of cinchona trees - can be used for treating COVID-19 patients. This anti-malarial also has broad-spectrum antiviral activity and regulatory effects on the immune system. Clinical evaluation of chloroquine phosphate in more than ten hospitals across several provinces in China has shown that it alleviates the symptoms for most patients and expedites virus seroconversion.

The epidemiologist Nanshan Zhong, who is credited with discovering the SARS coronavirus in 2003 and is advising on the management of the COVID-19 outbreak, has said that chloroquine phosphate is not a highly effective cure but its effects deserve attention, even though its pharmaceutical mechanism remains unclear. However, quinine and quinine derivatives have been used for two hundred years, and the bark from which it is extracted for far longer. Their safe usage and potential side effects are well established.

Another compound from herbal remedies recruited to control COVID-19 is diammonium glycyrrhizinate, an extract of liquorice roots. Liquorice, Glycyrrhiza glabra, has long been employed against coughs and colds as well as to settle disturbed digestion, while diammonium glycyrrhizinate has antiinflammatory activity and is used to treat liver damage caused by hepatitis B. Professor Hong Ding of Wuhan University has proposed a combination of diammonium glycyrrhizinate and vitamin C as a COVID-19 therapy. This approach became popular through social media and reporting in publications such as the Health Times (Jiankang Shibao). It has not been officially recommended, but clinical trials have recently been approved.

The rich tradition of herbal medicine in China is also being deployed against COVID-19. In the newest version of the diagnosis and treatment plan issued by the National Health of Commission of China, traditional Chinese medicine decoctions are explicitly recommended. Several patent herbal drugs, such as Huoxiang Zhengqi capsules, Lianhua Qingwen capsules and Radix isatidis granula, are being proposed as treatments, the latter two having also been used during the SARS-CoV outbreak in 2003. Boli Zhang, a leading traditional Chinese medicine expert advising on COVID-19 management, claims that such herbal medicines have been very useful in improving symptoms such as coughing, weakness and digestive system disorders as well as alleviating anxiety.

Compared to chemical drugs, herbal medicines and plant natural products are less understood mechanistically, but several clinical investigations have been started to more precisely evaluate their effects. For example, a project led by Nanshan Zhong aims to investigate the effects of Lianhua Qingwen on COVID-19. As Zhong says, drug development in Chinese medicine is largely based on experiences from clinical practices, which is philosophically different from the routine drug development strategy, and so may have several advantages.

In routine drug development, researchers first discover a drug molecule with potential therapeutic activity against a certain target, then optimize its structure and validate its function using in vitro experiments followed by animal and clinical trials. By contrast, many herbal drugs have been used in clinics for hundreds or thousands of years, and thus their safety and effects have been repeatedly tested; chloroquine phosphate has been used to treat malaria for over 70 years. Timeliness is another advantage, particularly during emergencies. Once a herbal decoction or component is found to be effective, it can be immediately used for treating patients, its safety already established.
Anti-viral herbal medicines have been used in many historic epidemics, for example the previous two coronavirus outbreaks (SARS-CoV in 2013 and MERS$\mathrm{CoV}$ in 2012), seasonal epidemics caused by influenza viruses and dengue virus. Extracts from Lycoris radiate, Artemisia annua and Lindera aggregate, and the natural products isolated from Isatis indigotica, Torreya nucifera and Houttuynia cordata, showed anti-SARS effects ${ }^{1-5}$. The plant flavone baicalein can prevent dengue virus entry into the host and inhibit post-entry replication ${ }^{6}$. Additionally, natural products from Pelargonium sidoides roots and dandelion have anti-influenza activities, as they inhibit virus entry and key viral enzyme activities ${ }^{7,8}$.

Like chloroquine phosphate, these herbal medicines are generally not highly potent and thus cannot be regarded as a cure. Nevertheless, as a complementary treatment they can elevate recovery rates when combined with other treatments. In an emergency like the current COVID-19 outbreak, drugs like remdesivir - an experimental drug developed against Ebola and recently held up by WHO Assistant Director-general Bruce Aylward as the only "drug right now that we think may have real efficacy" - take time to pass clinical trials, but readily available herbal medicines and natural products with proven safety can buy time as a first line of defence.

Plants are important not only for food but also for medicine. Understanding the taxonomy, ecology and conservation of herbs, as well as the pathways of secondary metabolite synthesis, is important for drug development. Investing in research into ethnobotany, phytochemistry, plant physiology and ecology will be vital in protecting the global population from current and future pandemics.

Published online: 13 March 2020 https://doi.org/10.1038/s41477-020-0628-0
References
1. Li, S. Y. et al. Antivir. Res. 67, 18-23 (2005).
2. Lin, C. W. et al. Antivir. Res. 68, 36-42 (2005).
3. Ryu, Y. B. et al. Bioorg. Med. Chem. 18, 7940-7947 (2010)
4. Yu, M. S. et al. Bioorg. Med. Chem. Lett. 22, 4049-4054 (2012).
5. Lau, K. M. et al. J. Ethnopharmacol. 118, 79-85 (2008).
6. Zandi, K. et al. BMC Complem. Altern. M. 12, 214 (2012).
7. Theisen, L. L. \& Muller, C. P. Antivir. Res. 94, 147-56 (2012).
8. He, W., Han, H., Wang, W. \& Gao, B. Virol. J. 8, 538 (2011). 